INPLASY

PROTOCOL

To cite: Zhou et al. Effects of exercise on Inflammatory Factors and IGF System in Breast Cancer Survivors: A Meta-analysis. Inplasy protocol 2021100101. doi:

10.37766/inplasy2021.10.0101

Received: 27 October 2021

Published: 27 October 2021

Corresponding author:

Meng Ding

dingmengpy@aliyun.com

Author Affiliation:

Shandong Normal University

Support: Shandong Normal University.

Review Stage at time of this submission: Formal screening of search results.

Conflicts of interest: None declared.

\section{Effects of exercise on Inflammatory Factors and IGF System in Breast Cancer Survivors: A Meta-analysis}

Review question / Objective: To explore the effects of exercise on IL-6, IL-10, IL-1 $\beta$, CRP, TNF- $\alpha$ and IGF-1, IGFBP-3 in patients with breast cancer, and use subgroup analysis to further explore the effects of different intervention time and mode on outcome indicators in order to determine the relationship between intervention period, intervention type and inflammatory factors, IGF system. To provide evidence for the hypothetical mechanism of exercise to prevent the development of breast cancer and improve its prognosis.

Information sources: PubMed, Embase, Web of Science, CNKI, Wanfang and VIP (China Science and Technology Journal) were systematically searched until April 2021. Search terms included "physical activity or exercise or sport or training" and "breast cancer or breast tumor or breast oncology" and "inflammatory or IL-6 or IL-10 or IL-1 $\beta$ or CRP or TNF-a or IGF or IGF-1 or IGFBP-3".

INPLASY registration number: This protocol was registered with the International Platform of Registered Systematic Review and Meta-Analysis Protocols (INPLASY) on 27 October 2021 and was last updated on 27 October 2021 (registration number INPLASY2021100101).

\section{INTRODUCTION}

Review question / Objective: To explore the effects of exercise on IL-6, IL-10, IL-1 $\beta$, CRP, TNF- $\alpha$ and IGF-1, IGFBP-3 in patients with breast cancer, and use subgroup analysis to further explore the effects of different intervention time and mode on outcome indicators in order to determine the relationship between intervention period, intervention type and inflammatory factors, IGF system. To provide evidence for the hypothetical mechanism of exercise to prevent the development of breast cancer and improve its prognosis.

Condition being studied: Breast cancer is the most common cancer among women all over the world, and it is also the most frequent cause of cancer death in women. Therefore, how to improve the survival rate of breast cancer patients and reduce the 
incidence of breast cancer is the main research content. At present, several mechanisms have been speculated about the etiology and progress of breast cancer, including inflammatory factors, IGF system and so on. The effect of chronic inflammation on tumorigenesis and tumor microenvironment is widely considered to play a key role in the risk of cancer occurrence, development and recurrence. Some studies have shown that systemic inflammation characterized by elevated TNF-a, IL-6 and CRP is associated with an increased risk of breast cancer progression and death. In addition, compared with the serum of patients with benign tumor, the expression of insulin-like growth factors-1(IGF-1) was higher in patients with breast cancer. Therefore, some scholars have proposed that the increase of IGF-1 level may indicate the progression and metastasis of tumor . In addition to IGF-1, insulin-like growth factor binding proteins (IGFBPs) can also be considered as part of the IGF system, which can indirectly regulate the activity of IGFs. A large number of studies have shown that IGF-1 is involved in the occurrence and development of tumors and is closely related to the prognosis of tumors. Insulinlike growth factor binding protein-3 (IGFBP-3) inhibits its anti-apoptotic effect in breast cancer cells by regulating the mitosis of IGFs. In view of the effect of inflammatory markers and IGF system on breast cancer, improving the inflammatory state and IGF system of breast cancer high-risk population may be a new way to reduce the risk of breast cancer. Studies have shown that inflammatory factors such as TNF- $a$, IL6, IL-10 and CRP are considered to be potential mediators between exercise and breast cancer. In addition, exercise is related to the levels of IGF-I and IGFBPs . Exercise can inhibit the IGF signal pathway by reducing the level of IGF-1. An increasing number of evidence shows that exercise can improve the levels of inflammatory factors and IGF system in breast cancer patients and reduce the risk of breast cancer patients. However, the point from individual studies is inconsistent. Sprod et al. found no significant changes in insulin-like growth factor binding protein-1 (IGFBP-1) and IGFPB-3 after 12 weeks of Taijiquan intervention in 21 breast cancer survivors. In addition, other meta-analyses have come to different conclusion. Therefore, this meta-analysis will focus on the effects of exercise on IL-6, IL-10, IL-1 $\beta$, CRP, TNF$\alpha$ and IGF-1, IGFBP-3 in patients with breast cancer, and use subgroup analysis to further explore the effects of different intervention time and mode on outcome indicators in order to determine the relationship between intervention period, intervention type and inflammatory factors, IGF system. To provide evidence for the hypothetical mechanism of exercise to prevent the development of breast cancer and improve its prognosis.

\section{METHODS}

Participant or population: Breast cancer survivors.

Intervention: Intervention measures: exercise intervention (including aerobic exercise, resistance exercise, resistance exercise combined with aerobic exercise, high-intensity interval training, etc.).

Comparator: Control group: routine nursing, placebo control.

Study designs to be included: Randomized controlled trial.

Eligibility criteria: (1) subjects: breast cancer survivors; (2) intervention measures:exercise intervention (including aerobic exercise, resistance exercise, resistance exercise combined with aerobic exercise, high-intensity interval training, etc.); (3) study content: effects of exercise group (physical activity) and control group on inflammatory factors or IGF system in patients with breast cancer; (4) intervention time: during the period of postoperative rehabilitation; (5) study type: randomized controlled trial and include at least one blank group.

Information sources: PubMed, Embase, Web of Science, CNKI, Wanfang and VIP (China Science and Technology Journal) 
were systematically searched until April 2021. Search terms included "physical activity or exercise or sport or training" and "breast cancer or breast tumor or breast oncology" and "inflammatory or IL-6 or IL-10 or IL-1 $\beta$ or CRP or TNF- $\alpha$ or IGF or IGF-1 or IGFBP-3".

Main outcome(s): The main outcome indicators included the levels of IL-6, IL-10, IL-1 $\beta$, CRP, TNF- $\alpha$, IGF-1 and IGFBP-3 in each group.

Quality assessment / Risk of bias analysis: The Cochrane manual evaluation standard (version 5.0.2) was used to comprehensively evaluate the literature quality, and objectively evaluate whether there were methodological errors and subjective biases. In summary, risk of bias was assessed in the following six domains: (1) Random sequence Generation; (2) Allocation Concealment; (3) Blinding; (4) Incomplete outcome data; (5) selective outcome reporting; (6) Other sources of bias. Use the above criteria to evaluate the quality of the article, and the results are judged by "low risk", "high risk" or "unclear risk".

Strategy of data synthesis: This study used stata14.0 and Rstudio 4.1.1 software to perform a meta-analysis of the differences between endpoint and baseline indicators (formula: SDchange= JSD12+SD22 $\left(2 * R^{\star} S D 1 * S D 2\right) R$ taken as 0.5$)$. Mean $\pm S D$ was chosen as the standard scale of effect in the article, and the statistics were expressed as weighted mean difference (WMD) with $95 \%$ confidence interval (Cl), P $<0.05$ as a statistically significant difference. The $I^{2}$ value was used to perform heterogeneity analysis among studies. when $I^{2}=0$, no heterogeneity among studies was considered, and a fixed-effects model was used; when $I^{2} \geq$ $50 \%$, heterogeneity among studies was found and a random-effects model was used.

Subgroup analysis: When there was heterogeneity between studies, subgroup analysis was used to analyze the sources of heterogeneity, such as grouping different intervention period and type for computational analysis of $\mathrm{I}^{2}$ values.

Sensitivity analysis: In order to increase the credibility of the meta-analysis, sensitivity analysis were conducted to analyze whether there was a significant effect of each article on the combinedresults.

Country(ies) involved: China.

Keywords: breast cancer, exercise, inflammatory factors, IGF system.

Contributions of each author:

Author 1 - Yanan Zhou.

Author 2 - Ningxin Jia.

Author 3 - Meng Ding. 